\title{
Estudio de correlación clínico-radiológica, sensibilidad y especificidad de ecotomografía transcraneana en enfermedad de Parkinson y otros trastornos del movimiento
}

\author{
PABLO VENEGAS F. ${ }^{1}$, GONZALO MIRANDA G. ${ }^{2}$, M. CONSUELO SILVA C. ${ }^{1}$
}

'Unidad de Trastornos del Movimiento, Departamento de Neurología y Neurocirugía. ${ }^{2}$ Servicio de Imagenología, Hospital Clínico Universidad de Chile. Santiago de Chile.

Recibido el 2 de marzo de 2010, aceptado el 16 de noviembre de 2010.

Este trabajo no tuvo fuentes externas de financiamiento.

Correspondencia: Dr. Pablo Venegas Francke Profesor Asistente de Neurología Universidad de Chile Los Talaveras 277, Ñuñoa Santiago de Chile Fono: $+56-2-9788260$ Fax: $+56-2-4177604$ E-mail: pablo.venegasf@ gmail.com

\section{Transcranial sonography for the diagnosis of Parkinson disease}

Background: The detection of hyperechogenicity of the substantia nigra using trans cranial sonography can be predictive of the diagnosis of Parkinson Disease. Aim: To report an experience with transcranial sonography for the diagnosis of Parkinson disease. Material and Methods: One hundred sixteen patients with movement disorders were subjected to a transcranial sonograpy to detect the presence of hyperchogenicity of the substantia nigra and basal ganglia. Afterwards, two physicians, unaware of the results of the sonography, examined the patients and reached a clinical diagnosis. The concordance between ultrasound results and the clinical diagnosis was analyzed. Results: In 64 patients, a clinical diagnosis of Parkinson disease was reached. Of these, 52 patients had substantia nigra hyperechogenicity and in 12, it was normal. On the other hand ultrasound was normal in 42 of 43 patients without a clinical diagnosis of Parkinson disease. Therefore the sensitivity and specificity of trans cranial ultrasound for the diagnosis of Parkinson disease was 81 and 97\%, respectively. Conclusions: Transcranial sonography has a good sensitivity and specificity for the diagnosis of Parkinson disease.

(Rev Med Chile 2011; 139: 54-59).

Key words: Doppler, transcranial, ultrasonography; Parkinson disease; Ultrasonography.
L

a tríada diagnóstica de bradicinesia, rigidez y temblor de reposo que caracteriza a la enamplio grupo de enfermedades, constituyendo el síndrome parkinsoniano o Parkinsonismo. En este síndrome se engloban la enfermedad de Parkinson idiopática, parkinsonismos secundarios y otras enfermedades degenerativas del sistema nervioso central (esporádicas o hereditarias), los llamados "síndromes Parkinson-Plus". De los pacientes con síndrome parkinsoniano cerca de $80 \%$ correspon- den clínicamente a una EP Idiopática ${ }^{1}$.

La EP es una entidad relativamente frecuente en los pacientes de edad avanzada, ubicada en el segundo lugar entre las enfermedades neurodegenerativas, después de la enfermedad de Alzheimer. Tiene una prevalencia e incidencia que muestran una marcada variación geográfica que varía en el caso de prevalencia entre 18/100.000 en China hasta 328/100.000 en India, con tasas de incidencia entre 4,5 y $21 / 100.000^{1.3}$. En nuestro país, la prevalencia alcanza 190/100.000 llegando al 1\% 
de la población mayor de 65 años ${ }^{4}$. La etiología del cuadro es desconocida, aunque se han postulado mecanismos genéticos y ambientales. Los primeros tendrían mayor influencia en cuadros de inicio precoz (antes de los 40 años) y los segundos en cuadros de origen más tardío.

La extensión del proceso patológico abarca locus ceruleus, núcleo olfatorio anterior, núcleo basal de Meynert, sustancia nigra, hipotálamo, corteza cerebral, núcleos motores de los nervios craneales y componentes centrales y periféricos del sistema nervioso autónomo.

La evolución neuropatológica de la EP ha sido caracterizada por los esposos Braak y en ella se detallan seis etapas, siendo la inicial la afección del bulbo olfatorio y médula oblongata ${ }^{5}$. En etapas más avanzadas existe degeneración de núcleos protuberenciales que participan en la regulación del tono muscular durante el sueño REM. Otras áreas comprometidas en la EP son los núcleos serotoninérgicos y catecolaminérgicos en general, cuya afección se expresa clínicamente por trastornos del ánimo y de ansiedad. El compromiso del núcleo dorsal del vago es responsable de la constipación ${ }^{5,6}$. Posterior a este compromiso progresivo y consecutivo, aparecen los síntomas motores de la enfermedad, momento en el cual la pérdida de neuronas dopaminérgicas en el SNC alcanza entre 50 y $70 \%$ y la disminución de las concentraciones de dopamina estriatal bordea el $60 \%$.

La EP es una enfermedad que se desarrolla lentamente a lo largo de los años de modo que en el momento del inicio de los síntomas motores han transcurrido varios años de muerte neuronal y adaptaciones neurofisiológicas y neuroquímicas que han evitado la aparición de dichas manifestaciones. La mayoría de los estudios con neuroimagen funcional (SPECT y PET) del estado dopaminérgico estriatal, así como los estudios postmortem, señalan un período preclínico de la enfermedad entre 4 y 13 años $^{8,9}$.

La ausencia de un marcador biológico de la enfermedad en vida hace que el diagnóstico de EP se base en criterios clínicos?. Si bien con una adecuada historia y examen físico el neurólogo podría llegar a lograr $90 \%$ o más de certeza diagnóstica, ésto lo logra una vez configurado el cuadro clínico motor, que se asienta en etapas más tardías del proceso neuro-degenerativo. Debido a esto, es fundamental contar con técnicas de apoyo diagnóstico en etapas tempranas de la enfermedad.
Se ha utilizado el SPECT para evaluar el transportador de dopamina, el cual permite diferenciar el temblor esencial del parkinsonismo, sin embargo, no diferencia claramente los distintos tipos de parkinsonismos degenerativos entre sí, por lo que tendría una utilidad limitada en el diagnóstico específico de $\mathrm{EP}^{10}$.

En los últimos quince años el uso de ecotomografía transcraneana (ECO-TC) en modo B ha sido una herramienta importante en el estudio diagnóstico y diagnóstico diferencial de los distintos trastornos del movimiento ${ }^{11}$. Dicha técnica permite evaluar la presencia de hiper-ecogenicidad de la sustancia nigra (SN), hallazgo frecuente en pacientes portadores de EP, siendo francamente patológico un área de hiperecogenicidad mayor o igual a $0,20 \mathrm{~cm}^{2}$ con indemnidad de los núcleos de la base (Figura 1). En experiencias de Alemania ${ }^{12,13}$ país donde inicialmente se desarrolló esta técnica, la sensibilidad es de $91 \%$ y especificidad de $96 \%$ para el diagnóstico de EP.

Si bien la causa de la hiperecogenicidad no es del todo conocida, tendría relación con la acumulación de fierro en dicha zona, elemento altamente ecogénico ${ }^{14}$.

La ecografía transcraneana también puede ayudar en el diagnóstico diferencial de otros síndromes parkinsonianos en particular el síndrome Parkinson-Plus y síndrome parkinsoniano secundario. Así, por ejemplo, esta técnica es útil en el diagnóstico de parálisis supranuclear progresiva (PSP) y degeneración córticobasal (DCB). En éste último cuadro, usualmente existe hipereco-

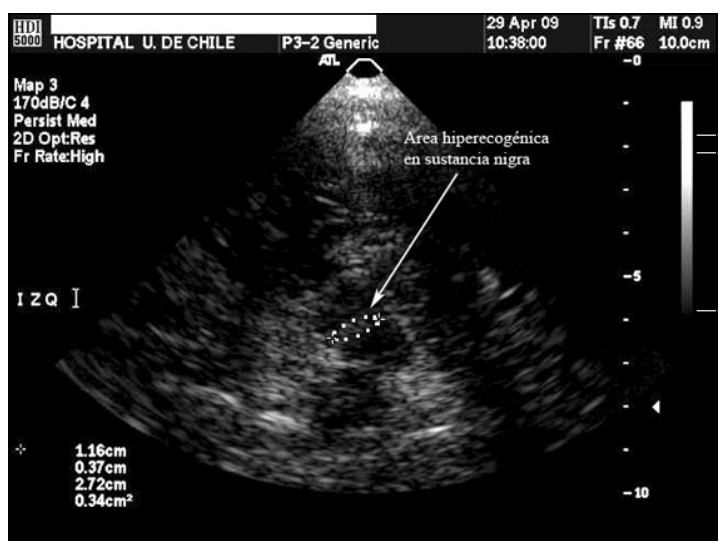

Figura 1. Visión ecográfica de mesencéfalo, donde se observa área de hiperecogenicidad patológica propia de enfermedad de Parkinson. 


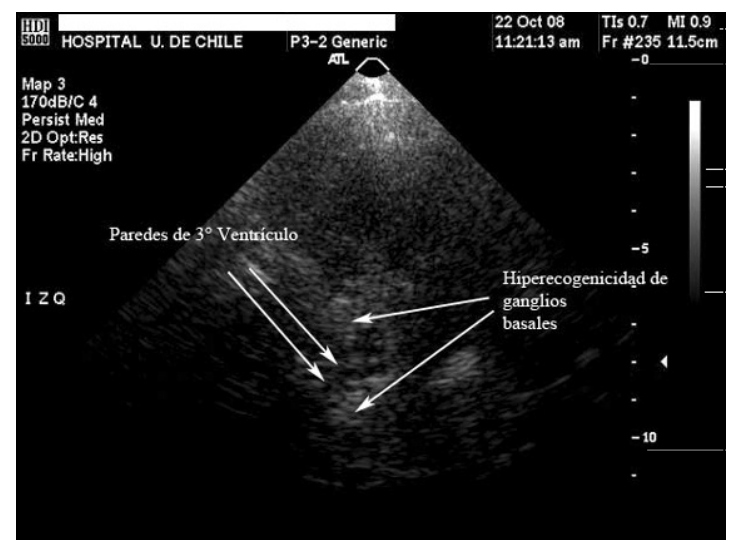

Figura 2. Visión ecográfica de tercer ventrículo y zona de ganglios basales. Nótese hiperecogenicidad de la zona de núcleos lenticulares.

genicidad tanto de la sustancia nigra como de los núcleos lenticulares, en tanto en PSP suele existir hiperecogenicidad únicamente de los núcleos lenticulares (Figura 2).

En casos de síndrome parkinsoniano secundario, el hallazgo ecográfico esperable es normalidad tanto de sustancia nigra como de ganglios basales. (Figura 3).

Otras características sonográficas, como ecogenicidad de núcleo del rafe mesencefálico, de núcleos rojos y núcleo lentiforme podrían ayudar en el diagnóstico diferencial de EP y otros trastornos del movimiento ${ }^{11}$.

El objetivo del presente trabajo es presentar la correlación clínico-radiológica de la ecografía transcraneana en una cohorte de pacientes con trastornos del movimiento evaluados en el Hospital Clínico de la Universidad de Chile, determinando valores de sensibilidad y especificidad de esta técnica para casos de EP, síndromes parkinsonianos secundarios y síndrome Parkinson-Plus.

\section{Pacientes y Método}

Se recluta una cohorte de 128 pacientes que son derivados a la Unidad de Trastornos del Movimiento del Departamento de NeurologíaNeurocirugía del Hospital Clínico de la Universidad de Chile entre septiembre del año 2008 y octubre de 2009.

Se les realiza ecografía transcraneana por ventana temporal con equipo sonográfico ATL

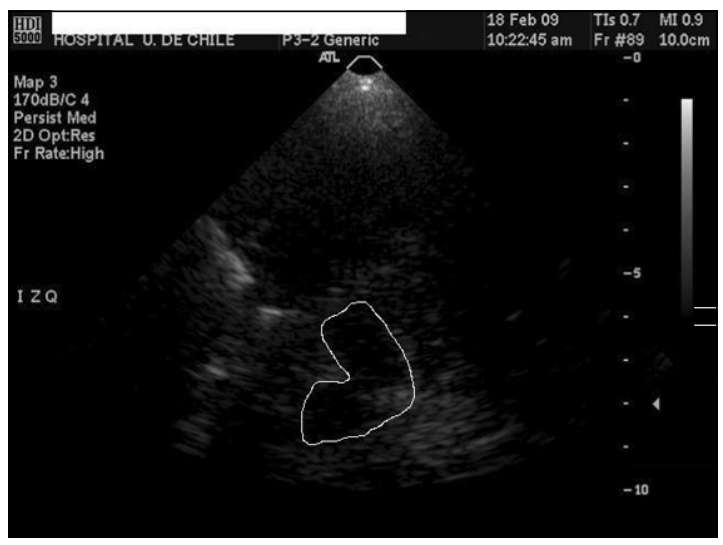

Figura 3. Visión normal de mesencéfalo propia de Parkinsonismo secundario. Nótese ausencia de ecogenicidad de sustancia nigra.

5000 , transductor de baja frecuencia $(2 \mathrm{~Hz})$ con el que se visualizan las estructuras mesencefálicas y ganglios de la base. Dicho examen es realizado por un neurólogo con formación y experiencia en ecografía transcraneana (PV) y un neurorradiólogo (GM) ciegos al diagnóstico de forma independiente. Se registra la presencia o ausencia de hiper-ecogenicidad de sustancia nigra y ganglios de la base, siendo positivo un tamaño mayor o igual a $0,20 \mathrm{~cm}^{2}$ para $\mathrm{SN}$ y positivo la sola presencia de hiperecogenicidad en el caso de ganglios de la base. Se realiza un informe por cada investigador y se evalúa la concordancia entre ambos. Luego se correlaciona dicho resultado con el diagnóstico clínico realizado a posteriori por los investigadores PV y CS.

En la determinación del diagnóstico se utilizan los criterios del Banco de Cerebros de la Sociedad de Parkinson del Reino Unido en el caso de enfermedad de Parkinson'; criterios de Louis et al para

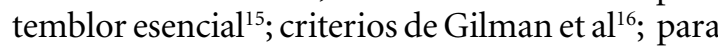
el diagnóstico de atrofia multisistémica y criterios del Consorcio de Demencia por cuerpos de Lewy para el diagnóstico de ésta ${ }^{17}$. En casos de parkinsonismo secundario (vascular, farmacológico) se utilizan antecedentes clínicos relevantes.

\section{Resultados}

De la cohorte de 128 pacientes, se obtiene diagnóstico clínico en 116 casos con una pérdida de seguimiento en 12 pacientes (10\%) (Tabla 1 ). 
Ecotomografía transcraneana en Parkinson - P. Venegas et al

Tabla 1. Diagnósticos clínicos. Se desglosan el número total y porcentaje de pacientes con diferentes diagnósticos

\begin{tabular}{|c|c|}
\hline Diagnóstico & $\begin{array}{c}\text { Número pacientes } \\
(\%)\end{array}$ \\
\hline Enfermedad de Parkinson (EP) & $64(55,17 \%)$ \\
\hline Parkinsonismo secundario & $18(15,52 \%)$ \\
\hline Temblor esencial (TE) & $16(13,79 \%)$ \\
\hline Parkinson-Plus & $9 \quad(7,8 \%)$ \\
\hline Sano & $3(2,59 \%)$ \\
\hline Síndrome atáxico & $2(1,72 \%)$ \\
\hline Depresión & $1 \quad(0,86 \%)$ \\
\hline Acatisia tardía & $1 \quad(0,86 \%)$ \\
\hline Demencia no especificada & $1 \quad(0,86 \%)$ \\
\hline Distonía & $1 \quad(0,86 \%)$ \\
\hline Total & 116 \\
\hline
\end{tabular}

Existe una concordancia de 100\% entre ambos investigadores (PV y GM) en cuanto a definir anormalidad de los hallazgos ecográficos.

La Tabla 2 muestra los hallazgos ecográficos, tanto a nivel de sustancia nigra como en ganglios de la base.

\section{Ecotomografía transcraneana en Enfermedad de Parkinson}

En esta cohorte de pacientes 64 tenían el diagnóstico clínico de EP. De ellos se encontró hiperecogenicidad patológica de $\mathrm{SN}$ en 52 casos (Verdaderos Positivos 52/64 =0,81), en 12 casos la SN fue normal (Falsos Negativos $12 / 64=0,18$ ). En solo 1 caso hubo alteración aislada de $\mathrm{SN}$ (hallazgo característico de EP) en el que el diagnóstico no fue EP o Parkinson-Plus (Falso Positivo $1 / 43=0,02$ ) y 42 casos en los cuales la SN fue normal y el diagnóstico no fue EP o Parkinson-Plus, diagnósticos en los cuales puede observarse alteración de SN (Verdadero Negativo 42/43 = 0.9767). La sensibilidad de ECO-TC para el diagnóstico de EP, fue por tanto de $81,25 \%$ y la especificidad de $97,67 \%$

\section{Ecotomogarfía transcraneana en Síndrome Parkinson-Plus}

En la cohorte de pacientes hubo nueve casos de Parkinson-Plus, cinco de los cuales correspondían a atrofia multisistémica (AMS), quienes tenían hiperecogenicidad de ganglios basales (GB) en el $100 \%$ de los casos e hiperecogenicidad en GB y SN en el $40 \% ; 3$ pacientes portadores de demencia por cuerpos de Lewy (DCL) con hiperecogenicidad de GB en el $100 \%$ de los casos e hiperecogenicidad

Tabla 2. Resultados ecográficos según patología

\begin{tabular}{|lrrrr|}
\hline Patología & \multicolumn{2}{c}{ Sustancia nigra } & \multicolumn{2}{c|}{ Ganglios basales } \\
Enfermedad de Parkinson (EP) & \multicolumn{1}{c}{$(+)$} & $(-)$ & \multicolumn{1}{c}{$(+)$} & $(-)$ \\
Temblor esencial (TE) & $52(81,25 \%)$ & $12(18,75 \%)$ & $1(1,6 \%)$ & $63(98,4 \%)$ \\
Parkinsonismo farmacológico & $0(0 \%)$ & $16(100 \%)$ & $0(0 \%)$ & $16(100 \%)$ \\
Parkinsonismo vascular & $0(0 \%)$ & $9(100 \%)$ & $0(0 \%)$ & $9(100 \%)$ \\
Atrofia multisistémica (AMS) & $0(0 \%)$ & $6(100 \%)$ & $0(0)$ & $6(100 \%)$ \\
Parkinsonismo secundario & $2(40 \%)$ & $3(60 \%)$ & $5(100 \%)$ & $0(0 \%)$ \\
Demencia por Cuerpos de Lewy (DCL) & $0(0 \%)$ & $3(100 \%)$ & $0(0 \%)$ & $3(100 \%)$ \\
Sano & $2(66,7 \%)$ & $1(33,3 \%)$ & $3(100 \%)$ & $0(0 \%)$ \\
Síndrome atáxico & $0(0 \%)$ & $3(100 \%)$ & $0(0 \%)$ & $3(100 \%)$ \\
Parkinson-Plus no especificado & $0(0 \%)$ & $2(100 \%)$ & $0(0 \%)$ & $2(100 \%)$ \\
Depresión & $0(0 \%)$ & $1(100 \%)$ & $1(100 \%)$ & $0(0 \%)$ \\
Acatisia tardía & $0(0 \%)$ & $1(100 \%)$ & $0(0 \%)$ & $1(100 \%)$ \\
Demencia no especificada & $0(0 \%)$ & $1(100 \%)$ & $0(0 \%)$ & $1(100 \%)$ \\
Distonía & $0(0 \%)$ & $1(100 \%)$ & $0(0 \%)$ & $1(100 \%)$ \\
\hline
\end{tabular}

Valor refleja el número total de pacientes. SN: Sustancia Nigra. GB: Ganglios de la base. 
de GB y SN en el 66,6\% de los casos y un paciente con Parkinson-Plus no especificado con resultado positivo para GB (100\%) y negativo para SN.

Por lo tanto, en el caso de síndrome ParkinsonPlus (AMS, DCL, Parkinson-Plus no especificado), el valor verdadero positivo, correspondiente a pacientes con enfermedad clínica y ecografía alterada, definiéndose ésta como la presencia de hiperecogenicidad de GB con o sin hiper-ecogenicidad de $\mathrm{SN}$, es de nueve pacientes y cero pacientes con enfermedad clínica y ecografía normal (falsos negativos). De los 107 pacientes restantes, 106 tuvieron ecografía negativa para Parkinson-Plus, es decir, sin ecogenicidad de GB (Verdaderos Negativos) y un paciente tuvo ecografía alterada (Falso Positivo), lo que da en nuestra experiencia una sensibilidad de $100 \%$ y una especificidad de $99,1 \%$ de la ECO-TC para el diagnóstico de Parkinson-Plus.

\section{Ecotomografía transcraneana en Síndrome Parkinsoniano Secundario:}

En esta cohorte, 18 pacientes corresponden a parkinsonismo secundario, incluyéndose causa farmacológica y vascular; nueve de ellos tienen Parkinsonismo farmacológico con resultado ecográfico negativo para GB y SN en el 100\% de los casos, 6 pacientes tienen Parkinsonismo vascular, en el $100 \%$ de los casos con resultados ecográficos negativos para SN y GB y en 3 pacientes no se especifica causa de Parkinsonismo secundario, con el $100 \%$ de los resultados ecográficos negativos para ambos. Por lo tanto, todos los pacientes con Parkinsonismo secundario tuvieron ecografía normal, hallazgo esperable en este cuadro (Verdaderos Negativos). Hemos mencionado que los hallazgos característicos de ECO-TC en Parkinsonismo secundario son los propios de un sujeto normal, por lo tanto en el contexto de población sana, el examen tiene baja especificidad; sin embargo, si lo acotamos a la población de pacientes con síndrome parkinsoniano la especificidad correspondería a 18/30 (60\%) donde 18 corresponde al número de verdaderos negativos y 30 a la totalidad de sujetos de la cohorte con síndrome parkinsoniano y que presentaban normalidad de SN y GB. Por lo tanto, en nuestra cohorte la sensibilidad de ECO-TC para Parkinsonismo secundario es $100 \%$ y la especificidad (considerando como población total a aquella que presenta un síndrome parkinsoniano) es de $60 \%$.

\section{Discusión}

Numerosos estudios han detectado hiper-ecogenicidad de la SN con indemnidad de los núcleos de la base en $\mathrm{EP}^{18-24}$, sin embargo, esta ecogenicidad patológica no está asociada con la severidad de la enfermedad y su tamaño no aumenta a medida que progresa la enfermedad ${ }^{25}$.

Los hallazgos ecográficos encontrados en este estudio confirman la utilidad de la ecografía transcraneana en el estudio diagnóstico de los distintos síndromes parkinsonianos.

Esta es la primera serie de pacientes a nivel nacional que analiza la sensibilidad de la técnica en distintos síndromes parkinsonianos. La sensibilidad de $81,3 \%$ para el diagnóstico de EP es levemente inferior a lo descrito en la literatura internacional, sin embargo, se encuentra en rangos aceptables para ser considerada un método confiable. Este resultado lo explicamos por el siguiente factor: los valores de referencia normales, esto es hasta $0,20 \mathrm{~cm}^{2}$ para $\mathrm{SN}$, han sido obtenidos en poblaciones distintas a la nuestra y con equipos ecográficos distintos; en nuestro centro hemos utilizado equipo ATL 5000 (Philips) mientras que los valores normales de series extranjeras, han sido obtenidos con equipos Siemens. La altísima especificidad para EP, obtenida en nuestra serie orienta a que el valor de corte para ecogenicidad normal de SN, utilizando nuestro equipo y en nuestra población, es menor a $0,20 \mathrm{~cm}^{2}$. Es probable que al utilizar un punto de corte más bajo la sensibilidad de nuestro equipo para diagnóstico de EP se eleve en torno a $90 \%$ con una especificidad algo menor a la obtenida actualmente y que esté en torno a $90 \%$, valores que replicarían a los obtenidos por centros de mayor experiencia.

En casos de Parkinson-Plus se encuentra una alteración de la ecogenicidad en el área de los núcleos de la base con indemnidad relativa de la sustancia nigra, y en el caso de los síndromes parkinsonianos secundarios no existirá alteración ni en sustancia nigra ni en núcleos de la base. En ambos grupos de Parkinsonismos obtuvimos una sensibilidad de $100 \%$ y una especificidad de $99,1 \%$ para Parkinson-Plus y 60\% para Parkinsonismo secundario, lo que avala la importancia de la ECOTC en el diagnóstico diferencial de estos cuadros en nuestro medio.

Por esto, concluimos que la ECO-TC es una herramienta útil, de alta sensibilidad y especifici- 
dad en el diagnóstico precoz y diferencial de EP. Nos parece de gran importancia que se potencie el desarrollo de esta técnica a nivel nacional para facilitar el diagnóstico diferencial de los distintos síndromes parkinsonianos.

\section{Referencias}

1. Chaná P, Kunstmann C y Tapia J. Enfermedad de Parkinson Idiopática. En Tratado de Neurología Clínica. Nogales-Gaete J., Donoso A., Verdugo R, editores. Santiago, Chile. Editorial Universitaria; año 2005, p. 483-90.

2. Hughes AJ. Clinic pathological aspects of Pakinson's disease. Eur Neurol 1997; 38 (Suppl 2): 13-20.

3. Caroline M, Tanner JP, Hubble P. Epidemiology and genetics of Parkinson's disease. En: Movement Disorders, neurologic principles and practice. Ray L. Watts, William C. Koller. Nueva York, Editorial Mc Graw-Hill; 2004, p. 138-39.

4. Chiofalo N, Kirschbaum A, Schoenberg B, Olivares O Soto E. Estudio epidemiológico de las enfermedades neurológicas en Santiago Metropolitano, Chile. Rev Neuro-Psiquiat 1992; 30: 355-41

5. Braak H, Del Tredici K, Rüb U, de Vos RA. Staging of brain pathology related to sporadic Parkinson's disease. Neurobiol Aging 2003. 24: 197-21.

6. Venegas P, Miranda G, Sinning M, Galleguillos L. Ecografía transcraneana para el diagnóstico de Enfermedad de Parkinson y otros trastornos del movimiento. Estudio Piloto de 39 casos y su correlato clínico-radiológico. Rev Hosp Clín Univ Chile 2009; 20: 5-13.

7. Morrish PK, Rakshi JS, Bailey DL, SawleGV, Brooks DJ. Measuring the rate of progression and estimating the preclinical period of Parkinson's disease with (18F) dopa PET. J. Neurol Neurosurg Psychiatry, 1998. 64(3): p.314-9.

8. Hilker R, Schweitzer K, Coburger S, Ghaemi M, Weissenbach S, Jacob AH, et al. Nonlinear progression of Parkinson Disease as determined by serial positron emission tomographic imaging of striatal fluorodopa $\mathrm{F}$ 18 activity. Arch Neurol 2005; 62: 78-82.

9. Hughes AJ, Daniel SE, Kilford L, Lees AJ. Accuracy of clinical diagnosis of idiopathic Parkinson's disease: a clinico-pathological study of 100 cases. J Neurol Neurosurg Psychiatry 1992; 55: 181-4.

10. Miranda M. Ecografía transcraneal: Una nueva herramienta útil en el diagnóstico de la enfermedad de Parkinson. Rev Med Chile 2006; 134: 525-8.
11. Berg D, Godau J, Walter U. Transcranial sonography in movement disorders. Lancet Neurol 2008; 7: 1044-55.

12. Behnke S, Berg D, Naumann M, Becker G. Differentiation of Parkinson's disease and atypical parkinsonian syndromes by transcranial ultrasound. J Neurol Neurosurg Psychiatry 2005; 76: 423-5.

13. Walter U, Dressler D, Wolters A, Probst T, Grossmann A, Benecke R. Sonographic discrimination of corticobasal degeneration vs progressive supranuclear palsy. Neurology 2004; 63: 504-9.

14. Miranda, M. Ecografía transcraneal como apoyo diagnóstico en la Enfermedad de Parkinson. Rev Chil NeuroPsiquiat 2007; 45: 76-7.

15. Louis E, Ford B, Lee H, Andrews H, Cameron G. Diagnostic criteria for essential tremor. Arch Neurol 1998; 55: 823-8.

16. Gilman S, Wenning G, Low P, Brooks D, Mathias C, Trojanowski J. Second consensus statement on the diagnosis of multiple system atrophy. Neurology 2008; 71: 670-6.

17. McKeith I, Dickson D, Lowe J, O Brien J, Feldman H, Cummings J. Diagnosis and management of dementia with Lewy bodies: third report of the DLB Consortium. Neurology 2005; 65: 1863-72.

18. Stockner H, Sojer M, KS K, Mueller J, Wenning G, Schmidauer C, Poewe W. Midbrain sonography in patients with essential tremor. Mov Disord 2007; 22: 414-7.

19. Ressner P, Skoloudik D, Hlustik P, Kanovsky P. Hyperechogenicity of the substantia nigra in Parkinson's disease. J Neuroimaging 2007; 17: 164-47.

20. Huang YW, Jeng JS, Tsai CF, Chen LL, Wu RM. Transcranial imaging of substantia nigra hyperechogenicity in a Taiwanese cohort of Parkinson's disease. Mov Disord 2007; 22: 550-5.

21. Walter U, Wittstock M, Benecke R, Dressler D. Substantia nigra echogenicity is normal in non-extrapyramidal cerebral disorders but increased in Parkinson's disease. J Neural Transm 2002; 109: 191-6.

22. Berg D, Siefker C, Becker G. Echogenicity of the substantia nigra in Parkinson's disease and its relation to clinical findings. J Neurol 2001; 248: 684-9.

23. Kolevski G, Petrov I, Petrova V. Transcranial sonography in the evaluation of Parkinson disease. J Ultrasound Med 2007; 26: 509-12.

24. Kim JY, Kim ST, Jeon SH, Lee WY. Midbrain transcranial sonography in Korean patients with Parkinson's disease. Mov Disord 2007; 22: 1922-6.

25. Berg D, Merz B, Reiners K, Naumann M, Becker G. Five-year follow-up study of hyperechogenicity of the substantia nigra in Parkinson's disease. Mov Disord 2005; 20: 383-5. 